\title{
Highly bright and photostable two-dimensional nanomaterials assembled from sequence-defined peptoids
}

Yang Song ${ }^{1,2, \$}$, Mingming Wang ${ }^{1, \sharp}$, Susrut, Akkineni ${ }^{1}$, Wenchao Yang ${ }^{1}$, Jeevapani J. Hettige $^{1}$, Haibao Jin ${ }^{1}$, Zhihao Liao ${ }^{1}$, Peng Mu ${ }^{1,3}$, Feng Yan, ${ }^{1,4}$ Marcel, Baer ${ }^{1}$, James J. De Yoreo ${ }^{1}$, Dan $\mathrm{Du}^{2}$, Yuehe Lin*2, and Chun-Long Chen*1,5

${ }^{1}$ Physical Sciences Division, Pacific Northwest National Laboratory, Richland, WA 99352, USA

2 School of Mechanical and Materials Engineering, Washington State University, Pullman, WA 99164, USA

${ }^{3}$ Department of Mechanical Engineering and Materials Science and Engineering Program, State University of New York, Binghamton, New York 13902, USA

${ }^{4}$ College of Chemistry \& Chemical Engineering, Linyi University, Linyi, Shandong 276005, China

${ }^{5}$ Department of Chemical Engineering, University of Washington, Seattle, WA 98195, USA.

${ }^{[\sharp]}$ These authors contributed equally to this work.

Correspondence and requests for materials should be address to C. Chen (Chunlong.Chen@pnnl.gov) or Y. Lin (yuehe.lin@wsu.edu) 


\section{Methods}

\section{Materials}

The $\beta$-alanine tert-butyl ester hydrochloride was purchased from Chem-Impex International, Inc. This hydrochloride salt was deprotected by adding a sodium hydroxide aqueous solution, then extracted with $\mathrm{CH}_{2} \mathrm{Cl}_{2}$, filtered and rotary evaporated for further reaction. N,N'-diisopropylcarbodiimide, Fmoc-6-aminohexanoic acid, bromoacetic acid and trifluoroactic acid (TFA) were purchased from Chem-Impex International, Inc. 2-(4-chlorophenyl)ethylamine and folic acid were purchased from VWR, Dansyl chloride was purchased from Acros Organics (part of Thermo Fisher Scientific Inc., CA, USA). F-12K Medium (GIBCO), Fetal bovine serum (GIBCO), Dextran (Alexa Fluor 647; 10,000 MW), CellLight Early Endosomes-RFP, Late Endosomes-RFP, BacMam 2.0, and LysoTracker Red DND-26 were purchased from Thermo Fisher., MA, USA. All other reagents were obtained from commercial sources and used without further purification.

\section{Synthesis of $\mathrm{Ncp}_{6} \mathrm{Nce}_{6}(\mathrm{Pep}-2)$ containing resins.}

Rink amide resins $(0.09 \mathrm{mmol})$ were used to generate $\mathrm{C}$-terminal amide peptoids. In the synthesis procedure, the Fmoc groups on the resins were deprotected by adding 2 $\mathrm{mL}$ of $20 \%(\mathrm{v} / \mathrm{v})$ 4-methylpiperidine/N,N-dimethylformamide (DMF), agitating for 40 minutes at room temperature for reaction, then filtering, and washing with DMF for 5 times. Then these resins were mixed with $1.5 \mathrm{~mL}$ DMF of $0.6 \mathrm{M}$ bromoacetic acid in DMF, followed by adding $0.30 \mathrm{~mL}$ of $50 \%$ (v/v) N,N-diisopropylcarbodiimide (DIC)/DMF. The mixture was agitated for 10 minutes at room temperature, then filtered and washed with DMF for 5 times. In the nucleophilic displacement step, 1.5 mL NMP (N-methyl-2-pyrrolidone) solution of primary amine monomers $(0.6 \mathrm{M})$, including 2-(4-chlorophenyl)ethylamine to generate $\mathrm{N}$-[2-(4-chlorophenyl)ethyl]glycine (Ncp) and $\beta$-alanine tert-butyl ester to generate $\mathrm{N}$-(2-carboxyethyl)glycine (Nce), were added into the above resins, followed by the agitation for 10 minutes at room temperature. After removing the excess monomer solution by filtration, the obtained resins were washed with DMF for 5 times. The acylation and displacement steps were repeated until Pep-2 peptoids were synthesized on resins.

\section{Synthesis of DNS-Pep-2 containing resins.}

Pep-2 containing rink amide resins $(0.09 \mathrm{mmol})$ were mixed with $0.9 \mathrm{mmol}$ dansyl chloride in $2 \mathrm{~mL}$ methanol. After adding $0.9 \mathrm{mmol} \mathrm{N}, \mathrm{N}$-Diisopropylethylamine into the above solution, the obtained mixture was stirred and reacted at room temperature for $12 \mathrm{~h}$. DNS-Pep-2 containing resins were obtained by five-time DMF washing and filtration.

\section{Synthesis of $\mathrm{Ncp}_{6} \mathrm{Nce}_{6} \mathbf{N c}_{6}(\mathrm{Pep}-3)$ containing resins.}

$0.9 \mathrm{mmol}$ Fmoc-6-aminohexanoic acid were dissolved in $1.5 \mathrm{~mL}$ DMF and $0.50 \mathrm{~mL}$ of $50 \%(\mathrm{v} / \mathrm{v}) \mathrm{N}, \mathrm{N}$-diisopropylcarbodiimide (DIC)/DMF were added in to the 0.09 
mmol Pep-2 containing resins. The mixture was agitated overnight at room temperature, then filtered, and washed well with DMF for 5 times. Fmoc groups were removed by adding $2 \mathrm{~mL}$ of $20 \%$ (v/v) 4-methylpiperidine/DMF solution, followed by a 40-minute agitation at room temperature. $\mathrm{Ncp}_{6} \mathrm{Nce}_{6} \mathrm{Nc}_{6}$ containing resins were obtained by a filtration and 5-time DMF washes to remove excess monomers and other reactants.

\section{Peptoid cleavage and HPLC purification}

The crude peptoid products were obtained by cleaving the corresponding resins with the addition of $95 \%$ trifluoroacetic acid (TFA) in water at room temperature. The suspension was filtered to get the crude peptoids in TFA solutions. TFA was then evaporated under a stream of $\mathrm{N}_{2}$ gas. Then, crude peptoids were dissolved in $\mathrm{H}_{2} \mathrm{O} / \mathrm{CH}_{3} \mathrm{CN}$ ( $\left./ \mathrm{v}=3: 7\right)$ for $\mathrm{HPLC}$ purification. All peptoids were purified by reverse-phase HPLC on an XBridge ${ }^{\mathrm{TM}}$ Prep $\mathrm{C} 18 \mathrm{OBD}^{\mathrm{TM}}$ column $(10 \mu \mathrm{m}, 19 \mathrm{~mm}$ $\times 100 \mathrm{~mm}$ ), using a narrow gradient of acetonitrile in $\mathrm{H}_{2} \mathrm{O}$ with $0.1 \%$ TFA over 15 $\min$.

\section{Peptoid sequences and their UPLC-MS characterizations}

Purified peptoids were analyzed using Waters ACQUITY reverse-phase UPLC (the corresponding gradient at $0.4 \mathrm{~mL} / \mathrm{min}$ over $7 \mathrm{~min}$ at $40^{\circ} \mathrm{C}$ with an ACQUITY®BEH C18, $1.7 \mu \mathrm{m}, 2.1 \mathrm{~mm} \times 50 \mathrm{~mm}$ column) connected with a Waters SQD2 mass spectrometry system. The final peptoid product was lyophilized from its solution in a mixture $(\mathrm{v} / \mathrm{v}=4: 6)$ of water and acetonitrile. The peptoid powder was finally divided into small portions $\left(1.0\right.$ or $\left.2.0 \times 10^{-6} \mathrm{~mol}\right)$ and stored at $-80^{\circ} \mathrm{C}$.

\section{Peptoid self-assembly}

Lyophilized peptoids were dissolved in the mixture of water and acetonitrile $(\mathrm{v} / \mathrm{v}=$ 1:1) to make $5.0 \mathrm{mM}$ clear solution, this clear solution was then transferred to $4{ }^{\circ} \mathrm{C}$ refrigerator for a slow evaporation. Gel-like or suspension materials containing a large amount of crystalline 2DNMs were obtained after a few days.

For the synthesis of Pep-fiber-67 nanofibers, a mixture of lyophilized DNS-Pep-2 $\left(3.33 \times 10^{-7} \mathrm{~mol}\right)$ and Pep-2 $\left(1.67 \times 10^{-7} \mathrm{~mol}\right)$ were in $0.25 \mathrm{~mL}$ water to first make a cloudy solution, a few drops of $1.0 \mathrm{M} \mathrm{NaOH}$ aqueous solution was then added to obtain a clear solution $(\mathrm{pH} \sim 9.0)$. The final solution $\mathrm{pH}$ was brought to $\mathrm{pH} 5.4$ by slowing adding drops of $1.0 \mathrm{M} \mathrm{HCl}$ aqueous solution and the obtained aqueous cloudy solution was then used for slow crystallization.

\section{AFM and TEM characterizations of peptoid 2D nanomembranes (2DNMs) and Pep-fiber-67 nanofibers}

AFM imaging (ex situ) was done in ScanAsyst mode at room temperature with a Bruker MultiMode 8. AFM samples were prepared by diluting peptoid assembly samples with water and using freshly cleaved mica as substrate.

TEM samples were prepared by pipetting one drop of water diluted peptoid assembly 
suspensions onto carbon-coated electron microscopy grid; $2 \%$ phosphotungstic acid was then used for negative staining. TEM measurements were conducted on a $200 \mathrm{kV}$ FEI Tecnai TEM microscope.

\section{Powder X-ray diffraction (XRD) measurement}

Powder XRD data were collected at a multiple-wavelength anomalous diffraction and monochromatic macromolecular crystallography beamline 8.3.1 at the Advanced Light Source located at Lawrence Berkeley National Laboratory (CA, USA). Beamline 8.3.1 has a $5 \mathrm{~T}$ single pole superbend source with an energy range of $5-17$ $\mathrm{keV}$. Data were collected with a $3 \times 3 \mathrm{CCD}$ array (ADSC Q315r) detector at a wavelength $(\lambda)$ of $1.1159 \AA$. Datasets were collected with the detector $200 \mathrm{~mm}$ from the sample. Peptoid sample suspensions or pellets were pipetted onto a Kapton mesh (MiTeGen) and dry. The XRD data was processed with custom Python scripts.

\section{Photoluminescent quantum yield (QY) measurement}

QY is considered as an important factor for quantitatively evaluating fluorescence intensity of materials, equals to the ratio of the number of emitted photons and the number of absorbed photons. In our experiment, a well-established reference method was employed for determining the QY value of 2DNMs as follow:

Quinine sulfate $\left(0.1 \mathrm{M} \mathrm{H}_{2} \mathrm{SO}_{4}\right.$; relative $\left.\mathrm{QY} \sim 0.58\right)$ was chosen as a reference. The QY of 2DNMs were calculated according to the following equation:

$$
\phi=\phi_{\mathrm{a}}^{\prime} \times \frac{\mathrm{A}_{\mathrm{a}}^{\prime}}{\mathrm{I}_{\mathrm{a}}^{\prime}} \times \frac{\mathrm{I}}{\mathrm{A}} \times \frac{\mathrm{n}^{2}}{\mathrm{n}_{\mathrm{a}}^{\prime 2}}
$$

Where $\phi$ is the QY of the 2DNMs, I is the integrated emission intensity of 2DNMs, ' $n$ ' and ' $A$ ' represent solvent refractive index and optical density, respectively. The superscript "," represents the reference data. For achieving the precious results, various concentrations of 2DNMs and referenced samples were adjusted the absorption below 0.10 (fluorescence cuvette).

\section{Photostability comparison of Cy5 dye, CdTe and CdSe/ZnS QDs, and 2DNMs.}

CdTe QDs and CdSe/ZnS QDs were commercial products (Sigma 900224-1ML; Sigma 777951-10MG). To guarantee a reliable comparison, the emission intensities of Cy5 dye, CdTe and CdSe/ZnS QDs, pre-assembled DNS-Pep-2 and 2DNMs were monitored at different time under a continuous UV irradiation using a $450 \mathrm{~W}$ xenon lamp. The fluorescence photobleaching curves of each samples were obtained by using spectrofluorimeter. Despite $\mathrm{CdTe}$ and $\mathrm{CdSe} / \mathrm{ZnS}$ QDs are photostable, their emission intensities almost completely disappeared after 15-minture UV irradiation.

\section{Time-resolved fluorescence Study.}

Time-resolved fluorescence measurements were performed with the time correlated, single-photon counting technique using the excitation pulses at $405 \mathrm{~nm}$ using Fluoromax-P (Horiba JOBIN YVON) photoluminescence spectrophotometer. The emission was collected through a polarizer set at the magic angle and an 8-nm band-pass monochromator (Jobin-Yvon H10) at $512 \mathrm{~nm}$. The instrumental response function was recorded with a polished aluminium reflector, and its full-width at halfmaximum was 40 ps. For time-resolved anisotropy measurements, the fluorescence 
decay curves were recorded at the vertical and horizontal positions of the excitation polarizer and with the emission polarizer set to the vertical position, and analyzed by the following equation:

$$
r(t)=\frac{I_{v}(t)-G \times I_{h}(t)}{I_{v}(t)+2 \times G \times I_{h}(t)}
$$

where $I_{v}$ and $I_{h}$ are the intensities collected at vertical and horizontal excitation polarizations, respectively, and $\mathrm{G}$ is the geometry factor at the emission wavelength, determined in independent experiments.

\section{Synthesis of nano-2DNMs}

To make micrometer-length 2DNMs suitable for intracellular study and cellular imaging, we cut them into small pieces to obtain nano-2DNMs-67 (2DNMs with nanometer-lengths) by ultra-sonication. In detail, the aqueous cloudy solution of 2DNMs was diluted with deionized water to have a concentration of $250 \mu \mathrm{M}$. This aqueous cloudy solution was then sonicated for 5 minutes with a sonicator probe at the power density of $120 \mathrm{~W}$ and with cooling of ice water. Nano-2DNMs were obtained by filtering sonication-cut 2DNMs through $0.22 \mu \mathrm{m}$ filter.

\section{Cell culture}

H1299 cells were purchased from the ATCC (VA, USA). Cells were cultured in F-12K Medium (MEM, GIBCO) with 10\% fetal bovine serum (FBS, Invitrogen, Life Technologies) in a $5 \% \mathrm{CO}_{2}$ incubator at $37{ }^{\circ} \mathrm{C}$. For imaging experiments, cells were seeded on Petri dish (Corning) at a density of $5 \times 10^{5}$ cells per dish and cultured overnight. Typically, H1299 cancer cells were incubated with nano-2DNMs-67 in F-12K medium containing 10\% FBS and then washed with PBS buffer (pH 7.4) before imaging.

\section{Cytotoxicity assay}

The cytotoxicity of nano-2DNMs-67, QDs and graphene nanosheets for H1299 cells in vitro was measured using the standard MTT assay. H1299 cells at a cell density of $1.0 \times 10^{5}$ cells $/ \mathrm{mL}$ were incubated in 96 -well plates for $24 \mathrm{~h}$. Then the medium was discarded and treated with F12K medium containing nano-2DNMs-67, QDs and graphene nanosheets at various concentrations for $24 \mathrm{~h}$. After that, the cells were incubated with $20 \mu \mathrm{L}$ of MTT $\left(5 \mathrm{mg} \cdot \mathrm{mL}^{-1}\right.$ in PBS) for $4 \mathrm{~h}$. After incubation, $150 \mu \mathrm{L}$ DMSO was added to each well. The optical density at a wavelength of $490 \mathrm{~nm}$ was recorded by a microplate reader.

\section{Photostability in live cell}

$\mathrm{H} 1299$ cells were pulsed at $37^{\circ} \mathrm{C}$ with concentrations of nano-2DNMs $(500 \mathrm{nM})$ in order to induce efficient endocytosis. Then H1299 cancer cells were incubated with staining solution of Dil Stain (V-22885, Life Technologies). The live cell images were acquired by Leica-TIRF-FRAP microscope using EM-CCD cameras for plasma membrane binding events and using spinning disk SD6000 confocal microscope for internalized particles. The nano-2DNMs were excited with a $405 \mathrm{~nm}$ laser, while the 
Dil dyes was excited with a $568 \mathrm{~nm}$ laser. Fluorescence images were analyzed using Fiji software (NIH). For each experiment, 2000 frames were acquired with frame rate of $300 \mathrm{~ms}$ each. The images were analyzed using Fiji software (NIH) and the fluorescence intensity of the tracks of these nano-2DNMs were calculated using Trackmate plugin post suitable thresholding.

\section{Photostability in fixed cell}

Briefly, for lysosome staining, H1299 cancer cells were cultured in $35 \mathrm{~mm}$ Petri dishes overnight. H1299 cancer cells were then incubated with staining solution of Cy5-phalloidin (Life Technologies) overnight, and then incubated with nano-2DNMs $(2 \mu \mathrm{M})$ for $4 \mathrm{~h}$ and chased for another $4 \mathrm{~h}$. The culturing supernatant of H1299 cells was removed and the cells were washed with $1 \times \mathrm{PBS}$ buffer $(\mathrm{pH} 7.4)$ three times. Finally, the cells were fixed with $4 \%$ paraformaldehyde (PFA) for $15 \mathrm{~min}$ and with DAPI for staining nuclei. The cell images were acquired by Leica-SP8 microscope. The nano-2DNMs were excited with a $405 \mathrm{~nm}$ laser, while the DAPI dyes were excited with a $360 \mathrm{~nm}$ laser and Cy5 dyes were excited with a $633 \mathrm{~nm}$ laser. Fluorescence images were analyzed using Fiji software (NIH). For each experiment, 4000 frames were acquired with frame rate of $300 \mathrm{~ms}$ each.

\section{Confocal imaging}

Briefly, for lysosome staining, H1299 cancer cells were cultured in $35 \mathrm{~mm}$ Petri dishes overnight. H1299 cancer cells were then incubated with staining solution of LysoTracker Red dyes (LysoTracker ${ }^{\circledR}$ Red DND-26, Life Technologies) overnight then incubated with nano-2DNMs $(2 \mu \mathrm{M})$ for $4 \mathrm{~h}$ and chased for another $4 \mathrm{~h}$. The culturing supernatant of H1299 cells was removed and the cells were washed with $1 \times$ PBS buffer ( $\mathrm{pH} 7.4)$ three times. Finally, these cells were fixed with 4\% PFA for 15 min and imaged using CLSM to observe co-localization of nano-2DNMs with lysosome. Confocal images were recorded with a Leica TCS SP8 confocal microscope. Nano-2DNMs-67 were excited with a $405 \mathrm{~nm}$ laser, while the LysoTracker Red dyes (LysoTracker ${ }^{\circledR}$ Red DND-26, Life Technologies) were excited with a $568 \mathrm{~nm}$ laser. Pearson's Correlation Coefficient (PCC) to represent the percentage of colocalized 2DNMs within lysosomes was recorded by the Leica Application Suite X (LAS X). Fluorescence images were analyzed using Fiji software (NIH). 
Table S1. Time-resolved fluorescence parameters of 2DNMs with different ratios of DNS-Pep-2.

\begin{tabular}{|l|l|l|l|l|l|l|}
\hline & $\tau_{\text {mean }}(\mathbf{n s})$ & $\boldsymbol{\tau}_{\mathbf{1}}(\mathbf{n s})$ & $\boldsymbol{\alpha}_{\mathbf{1}}(\mathbf{\%})$ & $\boldsymbol{\tau}_{\mathbf{2}}(\mathbf{n s})$ & $\boldsymbol{\alpha}_{\mathbf{2}}(\mathbf{\%})$ & QYs \\
\hline DNS-Pep-2 & 15.2 & 12.9 & 59.03 & 18.43 & 40.97 & 11.4 \\
\hline 2DNM-11 & 13.13 & 5.57 & 14.49 & 14.38 & 85.51 & 13.6 \\
\hline 2DNM-22 & 13.7 & 5.51 & 12.23 & 16.61 & 87.77 & 19.3 \\
\hline 2DNM-33* & 13.948 & 4.45 & 10.05 & 14.49 & 89.95 & 26.5 \\
\hline 2DNM-67 & 14.25 & 4.3 & 12.27 & 15.64 & 87.73 & 45.7 \\
\hline 2DNM-88 & 11.32 & 5.37 & 17.48 & 12.58 & 82.52 & 40.3 \\
\hline 2DNM-100 & 8.53 & 4.09 & 22.27 & 9.8 & 77.73 & 30 \\
\hline
\end{tabular}

*2DNM-11 represents the 2DNMs assembled from $11 \%$ of DNS-Pep-2 and $89 \%$ Pep- 2 . 2DNM-22 represents the 2DNMs assembled from 22\% of DNS-Pep-2 and 78\% Pep-2. 2DNM-33 represents the 2DNMs assembled from 33\% of DNS-Pep- 2 and $67 \%$ Pep- 2 .

$\tau_{1:}$ short lived decay component.

$\tau_{2:}$ long lived decay component.

$\boldsymbol{\alpha}_{1:}$ relative contribution of the lifetime component for $\tau_{1}$

$\boldsymbol{\alpha}_{2}$ : relative contribution of the lifetime component for $\tau_{2}$

The fluorescence decay may be modeled as the summation of multiple exponential components, as shown:

$$
F(t)=\alpha_{1} \times e^{-\frac{t}{\tau_{1}}}+\alpha_{2} \times e^{-\frac{t}{\tau_{2}}}
$$

Here $F(t)$ is the measured fluorescence intensity as a function of time $t ; \tau_{i}$ are the individual exponential components; and $\boldsymbol{\alpha}_{\mathrm{i}}$ are the coefficients of each exponential term. 

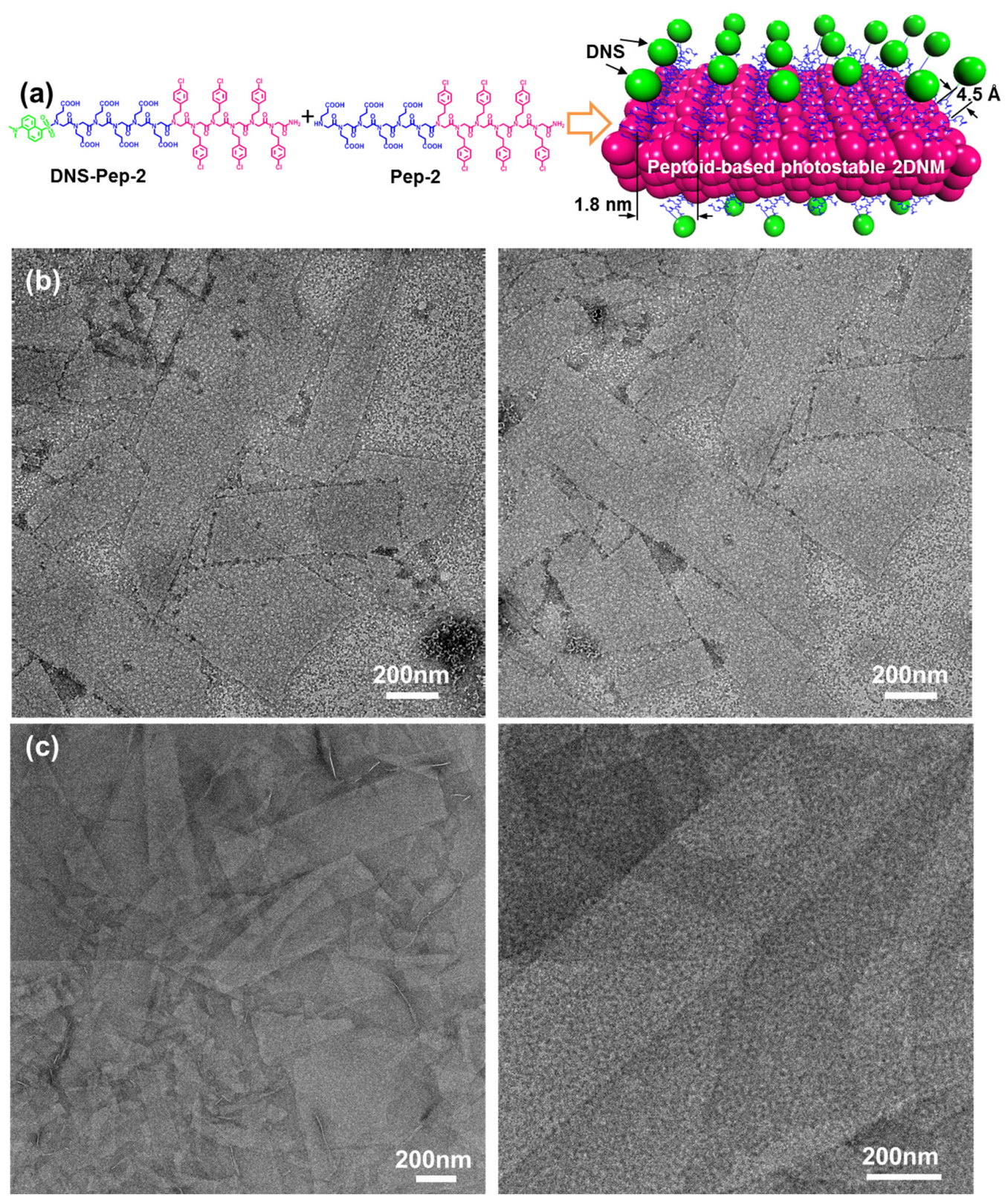

Figure S1. (a) Structures of DNS-Pep-2 and Pep-2, and a scheme showing their self-assembly into crystalline 2DNMs with precisely packed and aligned DNS dye molecules. The peptoid hydrophobic domains, polar domains, and dansyl (DNS) dyes are highlighted in red, blue, and green colors, respectively. (b) TEM images of 2DNMs co-assembled from DNS-Pep-2 and Pep-2 at the molar ratio of 1:2 (named 2DNM-33). (c) TEM images of 2DNMs assembled from DNS-Pep-2 (named 2DNM-100). 2.0\% phosphotungstic acid was used for negative staining. 


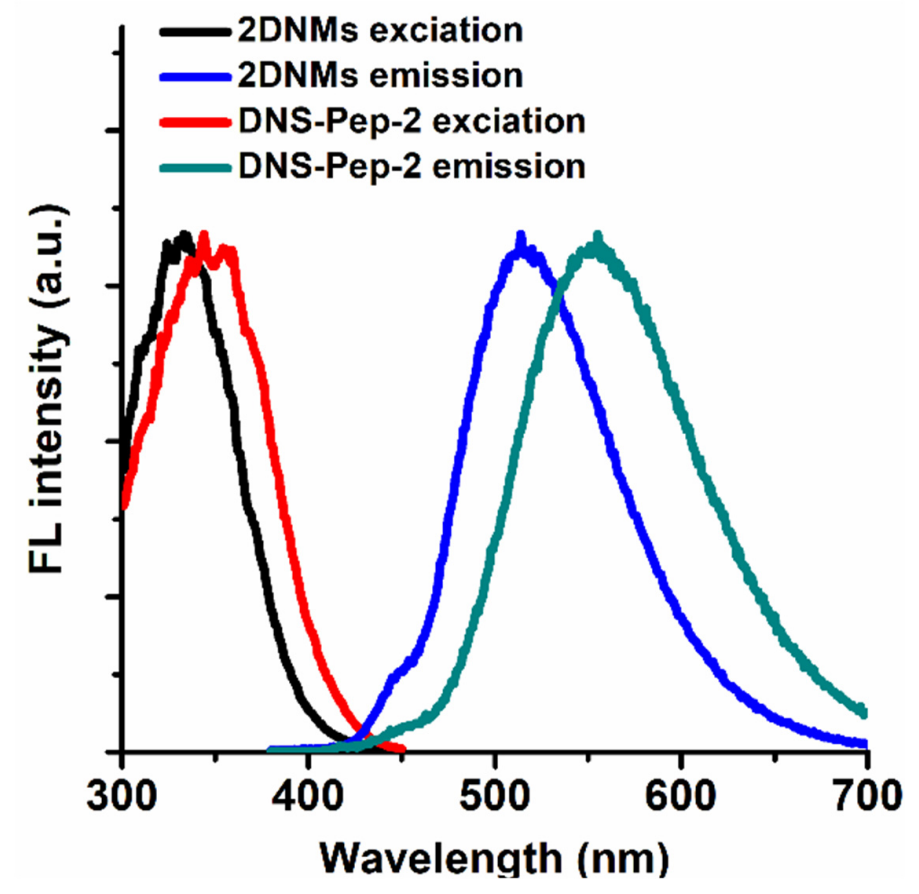

Figure S2. Fluorescence excitation and emission spectra of DNS-Pep-2 and 2DNMs assembled from DNS-Pep-2. 


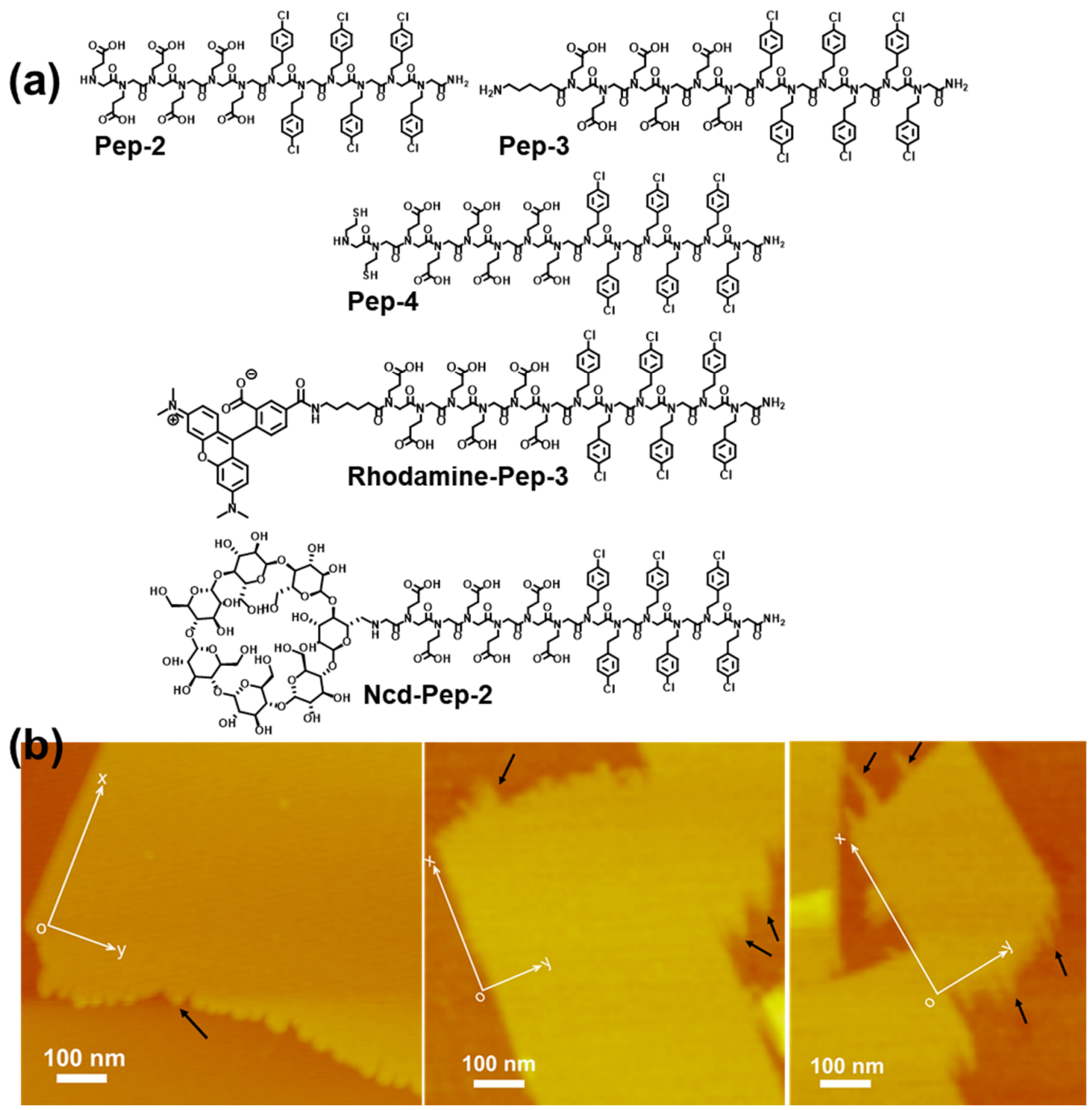

Figure S3. (a) List of five peptoids that were previously demonstrated for the repair or self-repair of membrane-mimetic nanosheets (2DNMs). ${ }^{1,2}$ (b) AFM images showing the overgrew edges of $2 \mathrm{DNM}-10 \mathrm{NH}_{2}$ co-assembled from DNS-Pep-2: Pep-2: Pep-3 $=4: 5: 1$. While edges of DNS-containing 2DNMs in the $y$ direction is straight, growth along $x$-direction was observed, indicating an anisotropic repairing process that is similar to that observed in the previous 2DNMs without DNS dyes. ${ }^{1,2}$ These AFM images were obtained by incubating pre-formed 2DNM sheets with aqueous solutions containing peptoid monomers and then being rinsed with water and dried for AFM imaging in the air. 

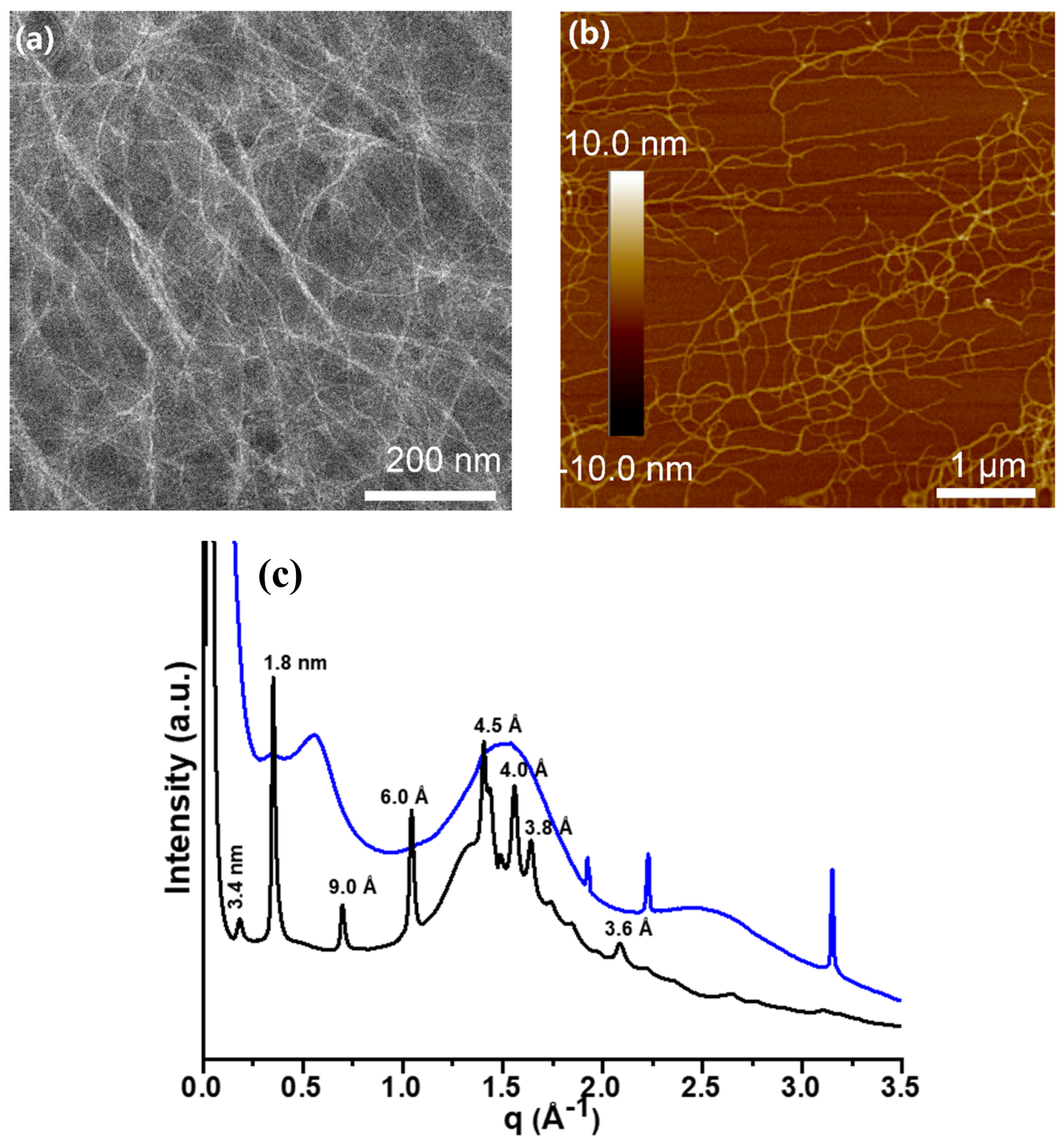

Figure S4. (a) TEM image of Pep-fiber-67 nanofibers assembled from 67\% of DNS-Pep-2 and 33\% Pep-2; 2.0\% phosphotungstic acid was used for negative staining. (b) AFM image of pep-fiber-67 nanofibers. (c) XRD data of Pep-2 2DNMs (Black) and pep-fiber-67 nanofibers (Blue) showing these pep-fiber-67 nanofibers are less crystalline and amorphous-like in contrast to 2DNMs. 


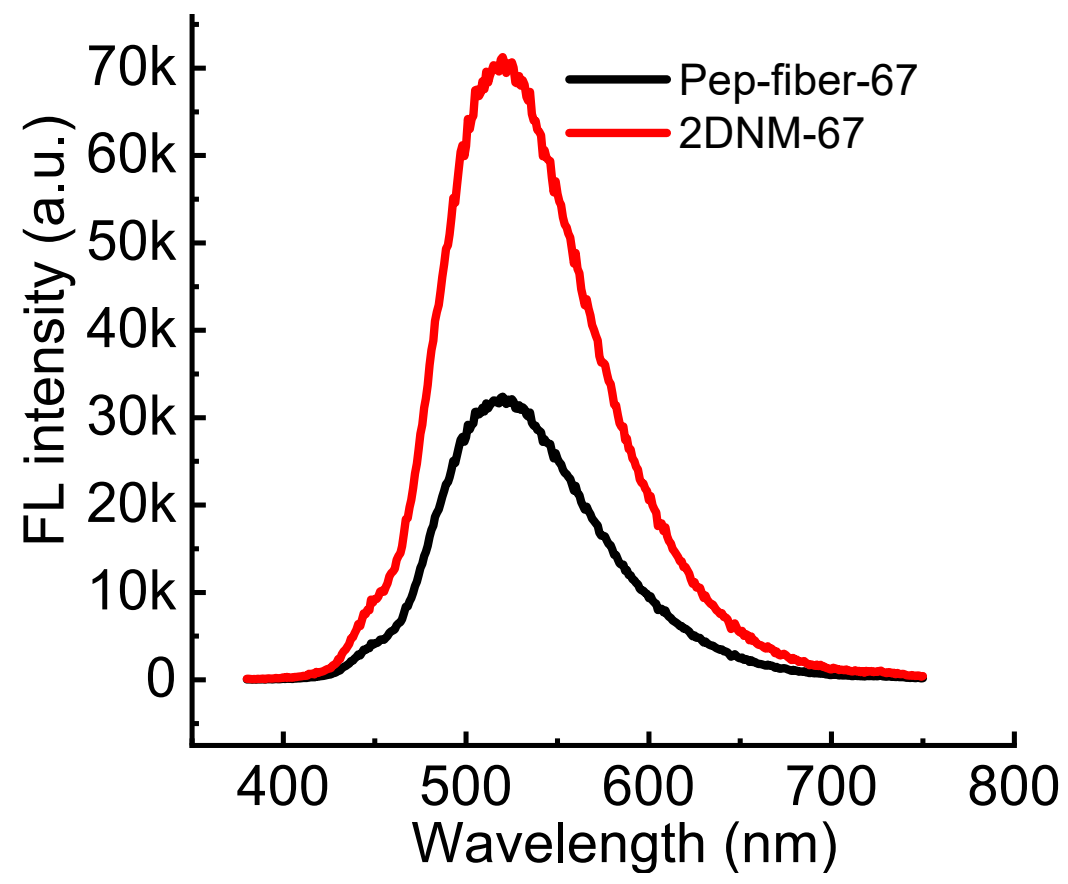

Figure S5. Florescence (FL) intensity of 2DNM-67 and Pep-fiber-67 in water. 

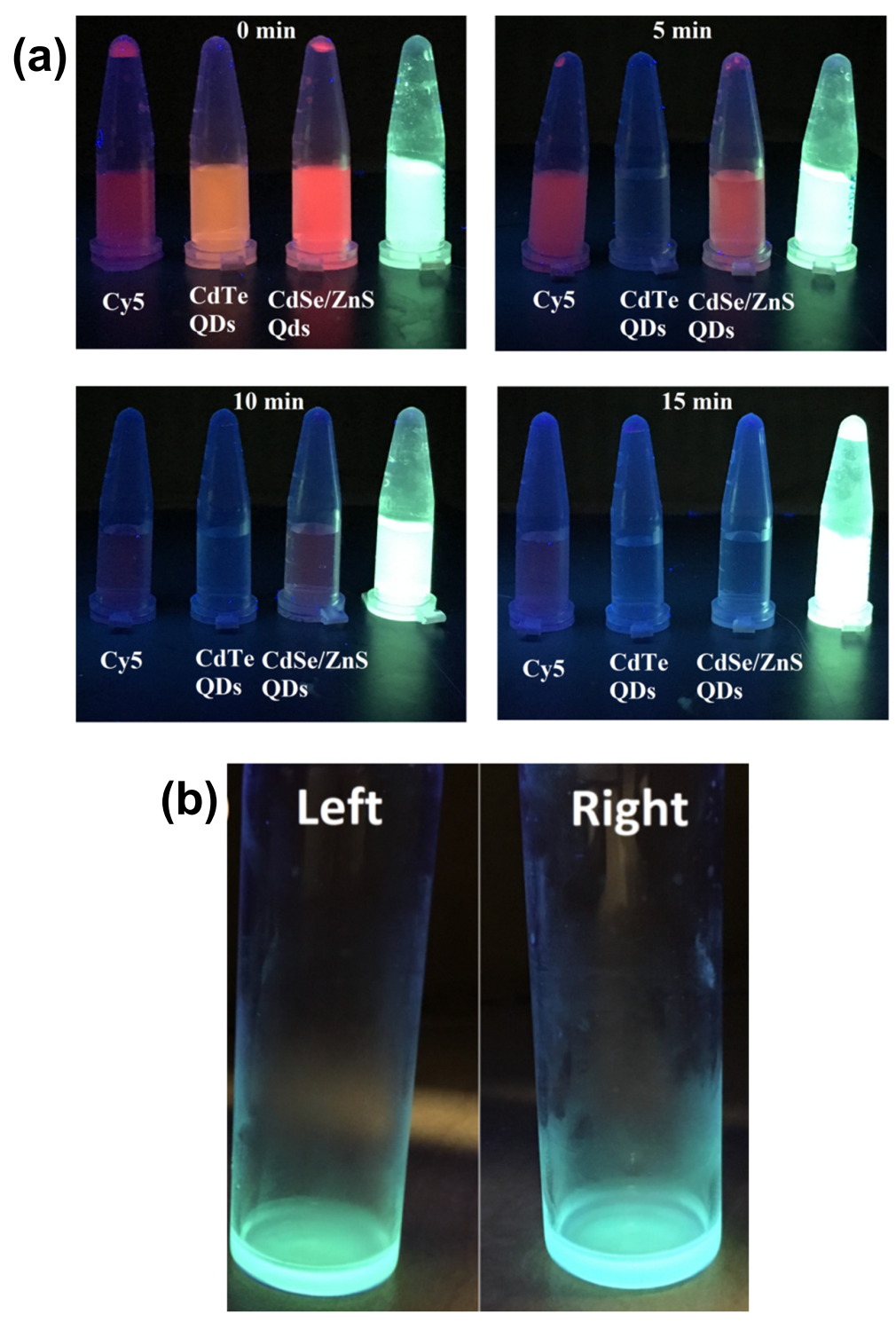

Figure S6. (a) Photographs of Cy5, CdTe and CdSe/ZnS QDs, and 2DNMs aqueous solution under UV irradiation for different time. All samples are continuously irradiated for $15 \mathrm{~min}$ by a $450 \mathrm{~W}$ xenon lamp. The concentrations of 2DNMs here are $10 \mu \mathrm{M}$. (b) Photographs of 2DNMs-67 dispersed in water before (left) and after (right) 3 months incubation. The concentration of $2 \mathrm{DNMs}$ is $10 \mu \mathrm{M}$. 

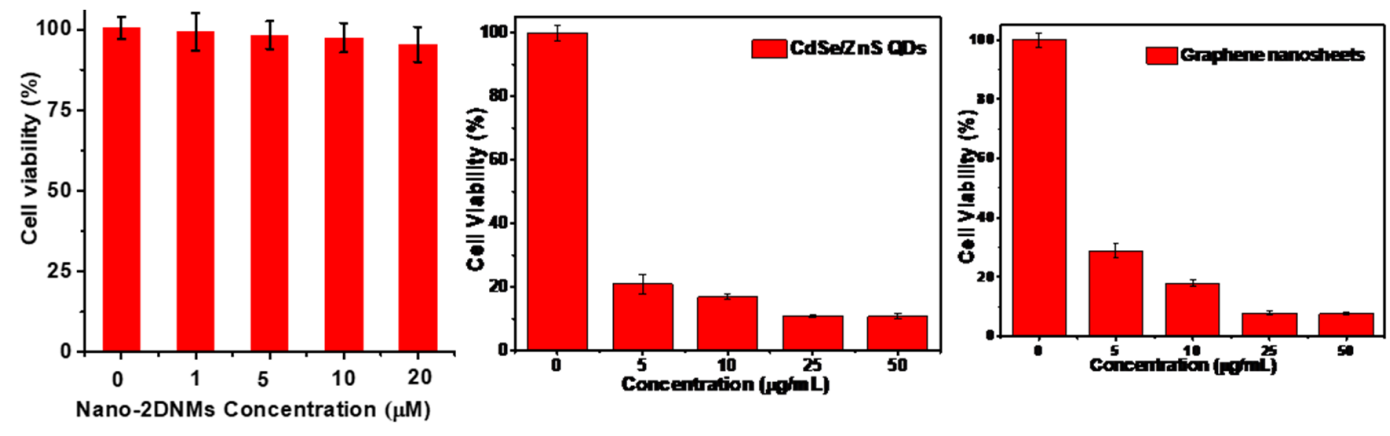

Figure S7. Results of MTT test for determining cytotoxicity of nano-2DNMs (left), $\mathrm{CdSe} / \mathrm{ZnS}$ quantum dots (QDs) (middle), and graphene nanosheets (right) by incubating them with $\mathrm{H} 1299$ cells at various sample concentrations. 

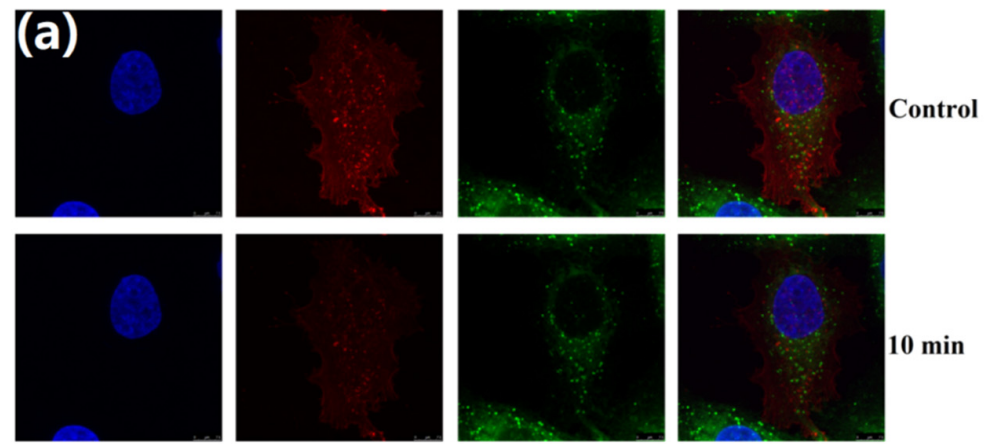

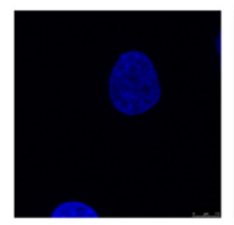

DAPI

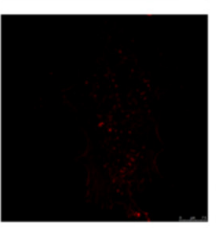

Cy5

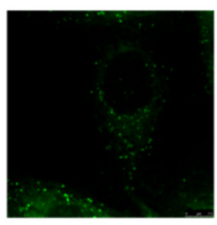

2DNMs

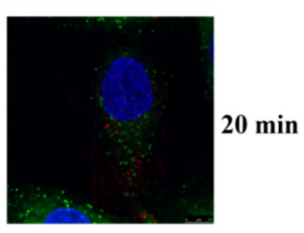

Overlap

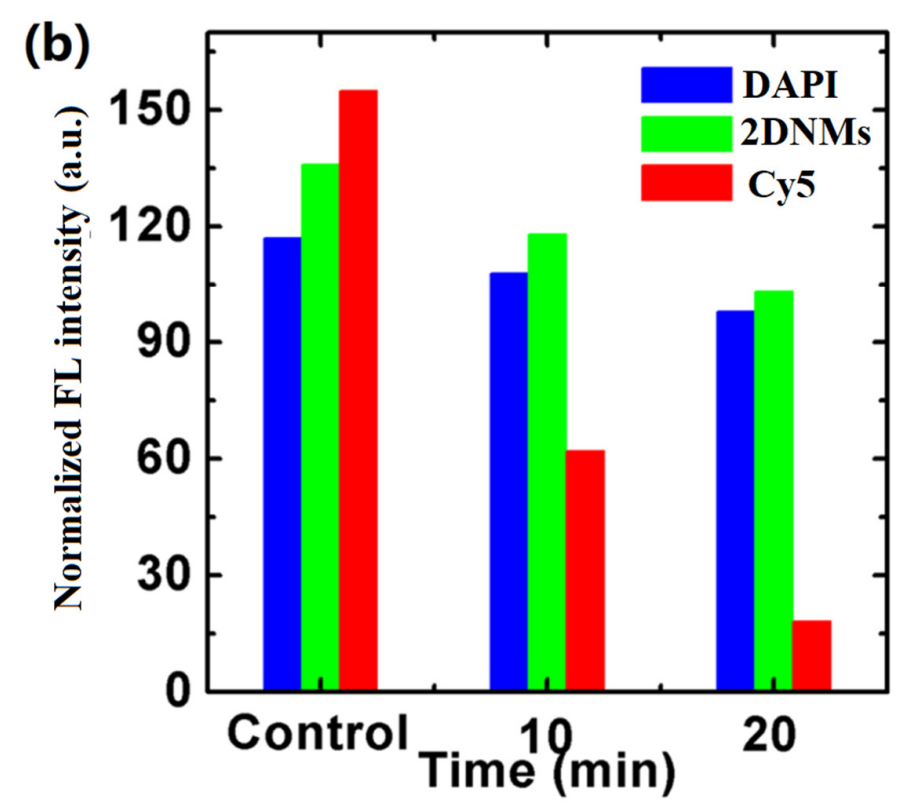

Figure S8. (a) A comparison of in-cell fluorescence stabilities of 2DNM-67 and Cy5-phalloidin conjugate in fixed H1299 cells. Repeated CLSM images of cells stained with DAPI (blue), 2DNM-67 (green) and Cy5 (red). H1299 cells were pulsed with respective 2DNM-67 at indicated concentrations, and the number indicates the number of frames. All images were acquired with images taken consecutively at 300 $\mu$ s intervals. (b) Normalized intracellular fluorescence intensity as a function of the number of recorded CLSM images. Normalization was carried out against the initial fluorescence intensity. 

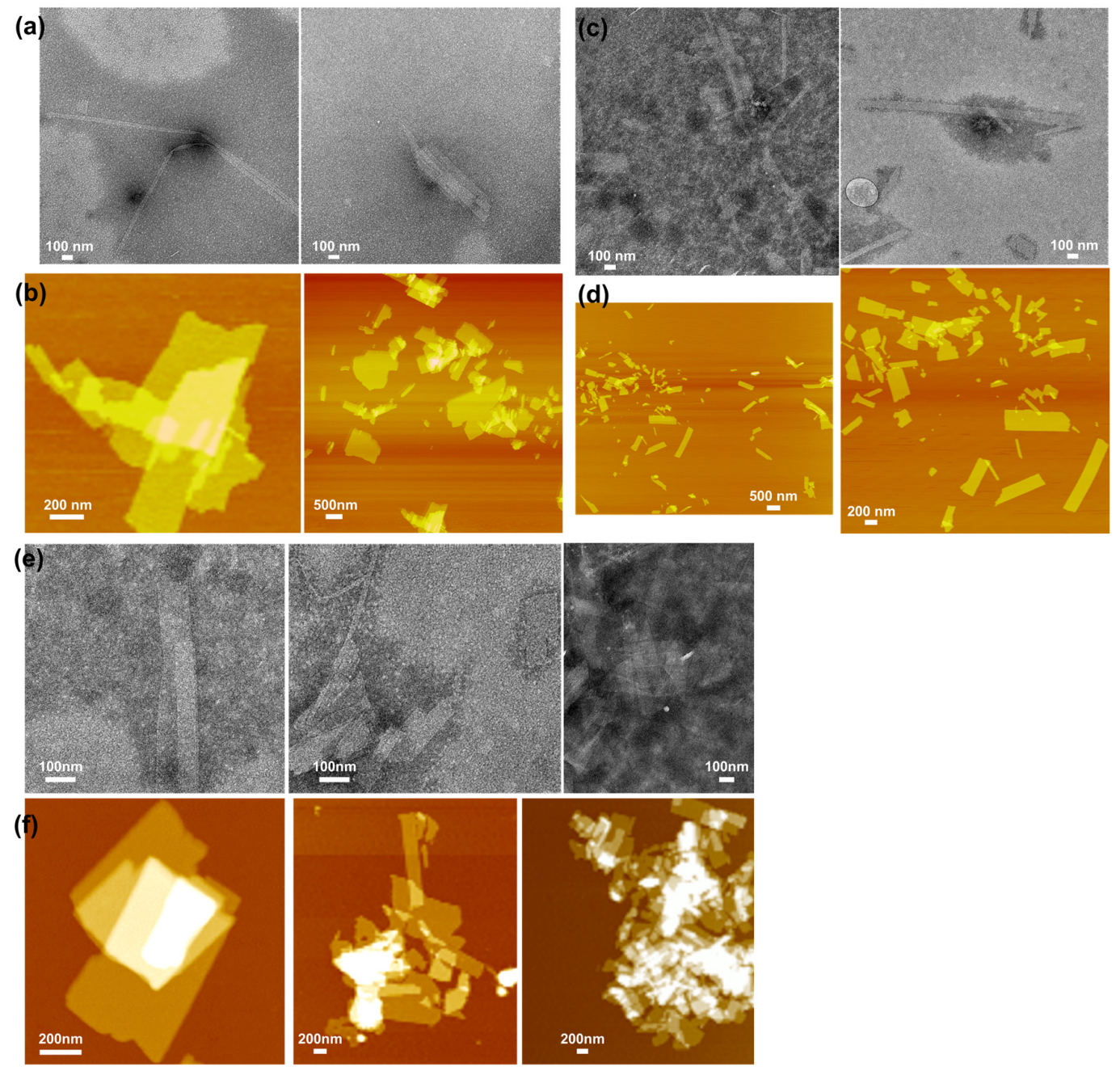

Figure S9. (a) TEM and (b) images of 2DNM-10NH $\mathrm{NH}_{2}$ co-assembled from DNS-Pep-2: Pep-2: Pep-3 = $4: 5: 1$. (c) TEM and (d) AFM images of 2DNM-30NH 2 co-assembled from DNS-Pep-2: Pep-2: Pep-3 = $4: 3: 3$. (e) TEM and (f) images of 2DNM-50NH 2 co-assembled from DNS-Pep-2: Pep-2: Pep-3=4: 1 : 5. 2.0\% phosphotungstic acid was used for negative staining for TEM imaging. 


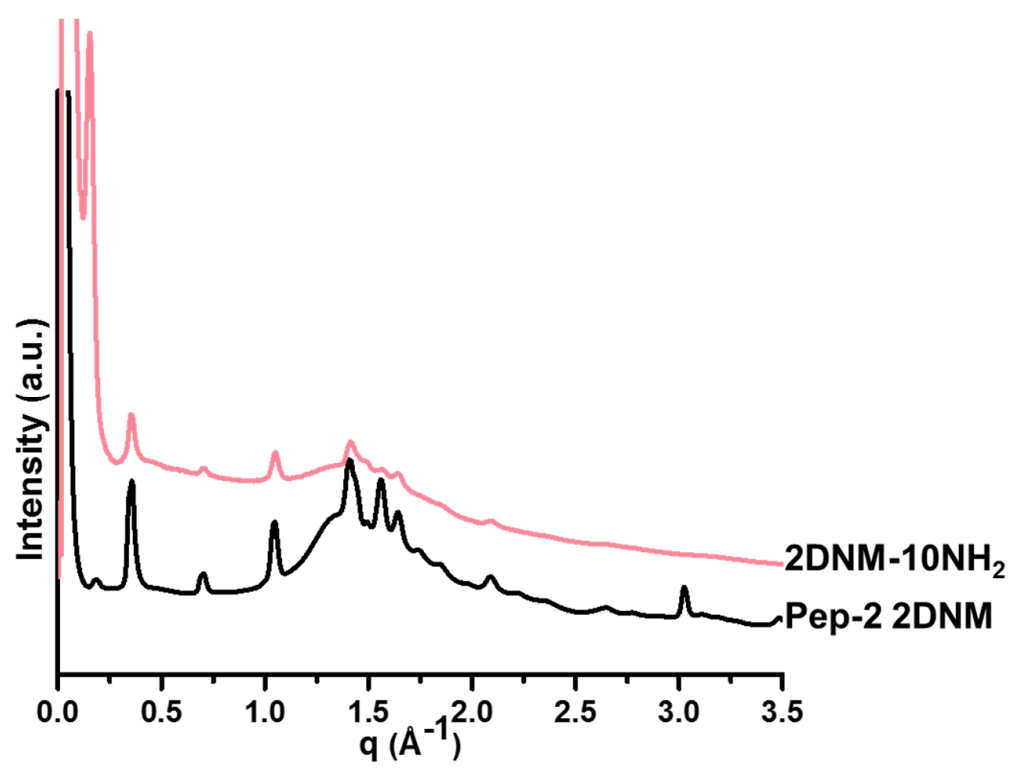

Figure S10. XRD data showing that 2DNMs assembled from DNS-Pep-2: Pep-2: Pep-3 in a ratio of 40: 50: 10 (named as $2 \mathrm{DNM}-10 \mathrm{NH}_{2}$ ) have a similar structure to 2DNMs assembled from Pep-2.

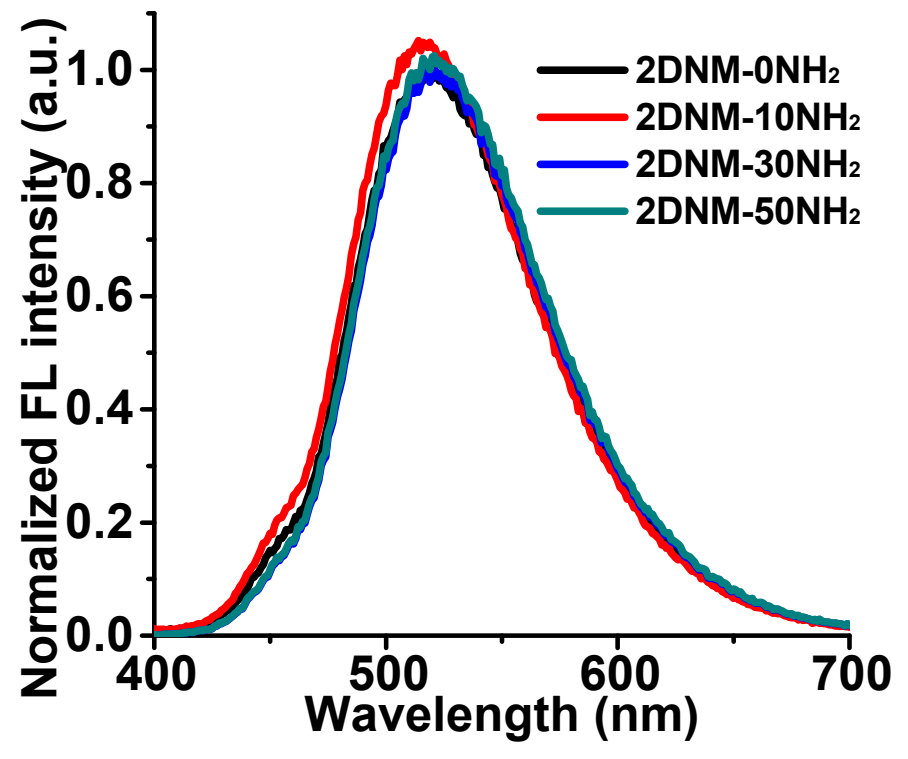

Figure S11. Normalized fluorescence (FL) intensity of 2DNMs with various number of amino groups, in which 2DNMs co-assembled from DNS-Pep-2: Pep-2 in a ratio of 40: 60 (named as 2DNM- $0 \mathrm{NH}_{2}$ ). 
Molecular Dynamics (MD) simulations: All simulations were performed using the GROMACS ${ }^{3-6}$ MD package. The system was generated using the CHARM-GUI ${ }^{7-9}$ web-based platform. The DNS-water system was modeled using all-atom Charmm36 ${ }^{10}$ force field. Geometry optimization was done on the initial system using steepest descent algorithm to remove any overlaps between atoms and hence minimize the energy of the system. Early equilibration of the system involved an NVT ensemble simulation followed by an NPT ensemble equilibration. Simulations use the same protocol as in Jin et al. ${ }^{1}$. The systems were simulated with periodic boundary conditions at $310 \mathrm{~K}$ temperature. In the NPT simulation runs, Nóse-Hooverlcite thermostat and Parinello-Rahman barostat were used to maintain the temperature of the system at $310 \mathrm{~K}$ and pressure at $1 \mathrm{~atm}$. The pressure was maintained at $1 \mathrm{~atm}$ in the NPT ensemble. The md integrator that utilizes the Leap-Frog algorithm was used for integrating Newton's equations of motion with a 2 fs time step. Cutoffs for the LJ and Coulombic interactions were set to $12 \AA$. Particle Mesh Ewald (PME) summation method with an interpolation order of 6 and $0.08 \mathrm{~nm}$ of FFT grid spacing was used for calculating the electrostatic interactions. Cutoffs for the LJ and Coulombic interactions were set to $12 \AA$.

Umbrella sampling simulations: To estimate the PMF, the umbrella sampling (US) was employed. Umbrella sampling ${ }^{11}$ is an enhanced sampling technique used to sample the configurational space more efficiently. In this method, a biasing potential is applied to the real interaction potential (in here, the interaction between the two dansyl dyes) causing the system to accurately sample the phase space. The reaction coordinate for the biased sampling is the distance between the $\mathrm{N}$ atoms (adjacent to the $\mathrm{S}$ atom) of the two dansyl dyes. The initial 112 structures were taken from a pulling simulation along the reaction coordinate. In here, the second dansyl dye was pulled away from the first with a $1000 \mathrm{~kJ} \mathrm{~mol}^{-1}$ pulling force and at a $0.01 \mathrm{~nm} \mathrm{ps}{ }^{-1}$ pulling rate. Each window was equilibrated for $100 \mathrm{ps}$ in the NPT ensemble prior to US. Each window was run for $1 \mathrm{~ns}$ in the US simulation setup. The potential of mean force (PMF) for the interaction between the two DNS molecules was reconstructed using the Weighted Histogram Analysis Method (WHAM) as implemented in GROMACS tools (gmx_wham). ${ }^{12}$

Umbrella potential

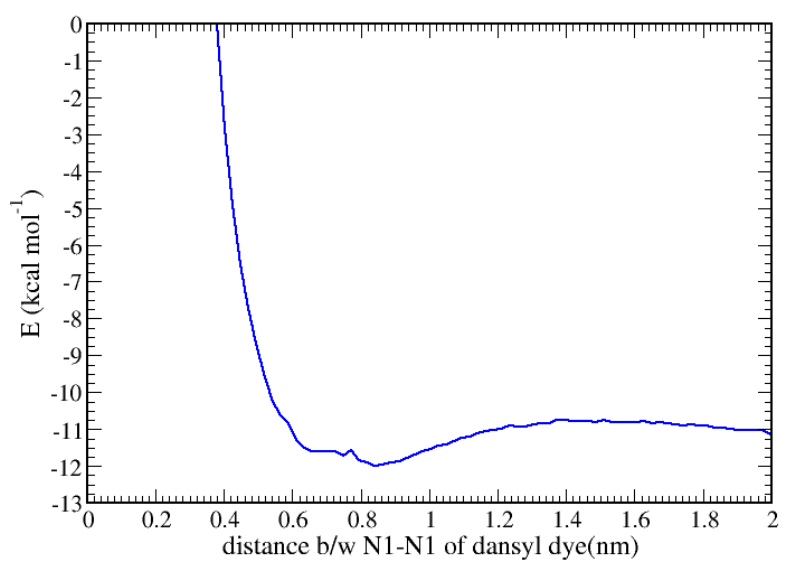


Figure S12. DNS-DNS Dimerization free-energy in water.

The dimerization free-energy between to DNS is about $1.5 \mathrm{kcal} / \mathrm{mol}$ for a the $\mathrm{N}$ to $\mathrm{N}$ distance, with a minimum around $0.9 \mathrm{~nm}$. This corresponds to the most likely $\mathrm{N}$-terminal distance in 2DNM as calculated free membrane simulations following the same protocol as in Jin et al. ${ }^{1}$ Analysis of the dimer region reveals multiple possible arrangements. The distance between center the aromatic rings and the angle between the normal vectors of the aromatic system can be used to better understand the sampled orientations.

The 2D free energy is estimated from the ensemble sampled in the biased simulations and shown in Fig S12. For close contacts of the aromatic rings the most likely is a T-shaped perpendicular arrangement but also parallel displaced arrangements are thermally accessible. ${ }^{13,14}$ Many configurations cannot be categorized as typical $\pi-$ $\pi$ interactions. Clearly, the formation of the dimer changes dynamical behavior of the dye and the available conformations, the underlying mechanism of adduct induced emission or crystallization induced emission. More detailed studies investigating the effect of crowding and the templating of the 2DNM are necessary in the future.

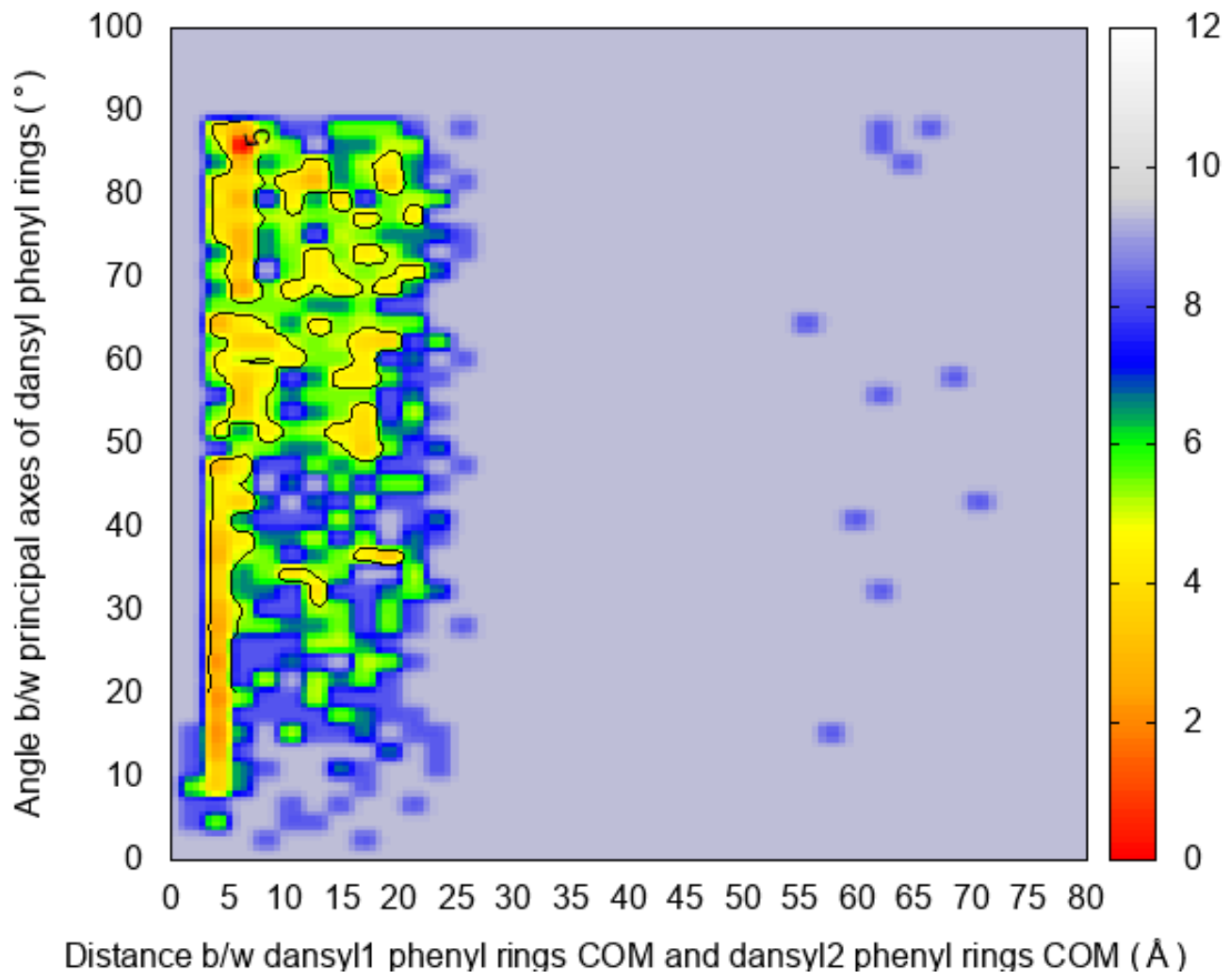

Figure S13. 2D-Free energy calculated from the joint probability distribution of the center of mass distance between the dye aromatic rings and the angle between the normal of the aromatic ring. Angles around $80^{\circ}$ correspond to T-shaped perpendicular orientations and around $20^{\circ}$ are parallel displaced arrangements 


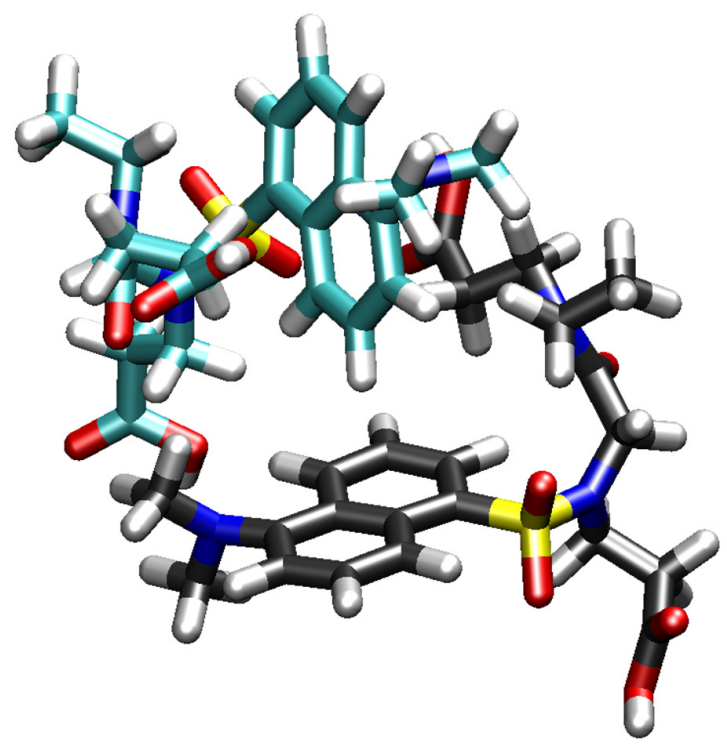

Figure S14. Representative conformation of a dimer in a T-shaped perpendicular orientation from the simulation. Carbon in cyan and black for dye one and two respectively. Oxygens are shown in red, nitrogen in blue, sulfur in yellow and hydrogens in silver. 

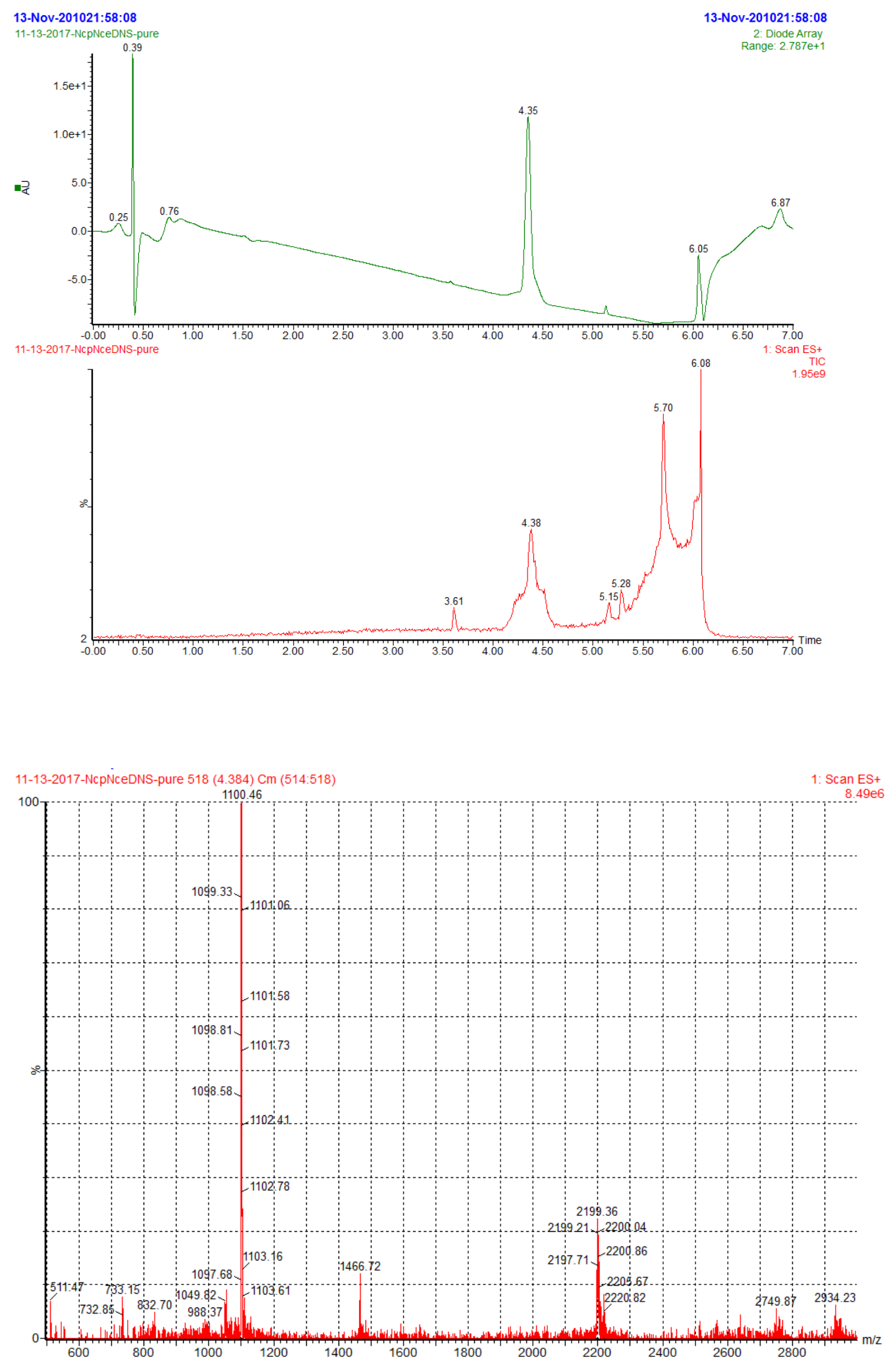

Figure S15. UPLC and MS data of DNS-Pep-2 peptoid. 


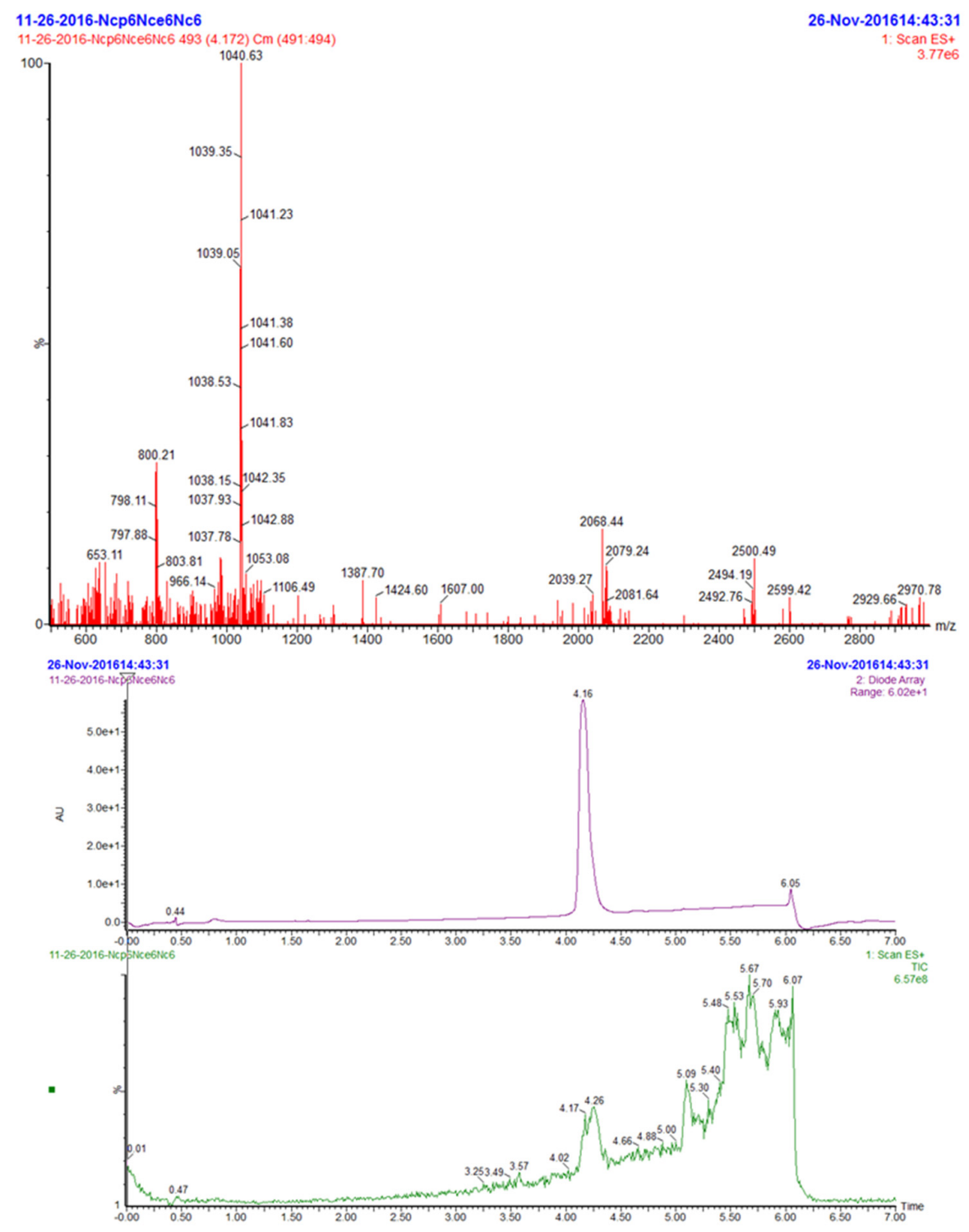

Figure S16. MS and UPLC data of Pep-3 peptoid.

\section{References:}

1. Jin, H.; Jiao, F.; Daily, M. D.; Chen, Y.; Yan, F.; Ding, Y.-H.; Zhang, X.; Robertson, E. J.; Baer, M. D.; Chen, C.-L. Highly stable and self-repairing membrane-mimetic 2D nanomaterials assembled from lipid-like peptoids. Nat. Commun. 2016, 7, 12252.

2. Jiao, F.; Chen, Y.; Jin, H.; He, P.; Chen, C.-L.; De Yoreo, J. J. Self-repair and patterning of 2D membrane-like peptoid materials. Adv. Funct. Mater. 2016, 26, 8960-8967. 
3. Berendsen, H. J. C.; van der Spoel, D.; van Drunen, R. GROMACS: A message-passing parallel molecular dynamics implementation. Comput. Phys. Commun. 1995, 91, 43-56.

4. Hess, B.; Kutzner, C.; van der Spoel, D.; Lindahl, E. GROMACS 4: Algorithms for Highly Efficient, Load-Balanced, and Scalable Molecular Simulation. J. Chem. Theory Comput. 2008, 4, 435-47.

5. Lindahl, E.; Hess, B.; van der Spoel, D. GROMACS 3.0: a package for molecular simulation and trajectory analysis. J. Mol. Model. 2001, 7, 306-317.

6. Van Der Spoel, D.; Lindahl, E.; Hess, B.; Groenhof, G.; Mark, A. E.; Berendsen, H. J. GROMACS: fast, flexible, and free. J. Comput. Chem. 2005, 26, 1701-18.

7. Brooks, B. R.; Brooks, C. L., 3rd; Mackerell, A. D., Jr.; Nilsson, L.; Petrella, R. J.; Roux, B.; Won, Y.; Archontis, G.; Bartels, C.; Boresch, S.; Caflisch, A.; Caves, L.; Cui, Q.; Dinner, A. R.; Feig, M.; Fischer, S.; Gao, J.; Hodoscek, M.; Im, W.; Kuczera, K.; Lazaridis, T.; Ma, J.; Ovchinnikov, V.; Paci, E.; Pastor, R. W.; Post, C. B.; Pu, J. Z.; Schaefer, M.; Tidor, B.; Venable, R. M.; Woodcock, H. L.; Wu, X.; Yang, W.; York, D. M.; Karplus, M. CHARMM: the biomolecular simulation program. J. Comput. Chem. 2009, 30, 1545-614.

8. Jo, S.; Kim, T.; Iyer, V. G.; Im, W. CHARMM-GUI: a web-based graphical user interface for CHARMM. J. Comput. Chem. 2008, 29, 1859-65.

9. Lee, J.; Cheng, X.; Swails, J. M.; Yeom, M. S.; Eastman, P. K.; Lemkul, J. A.; Wei, S.; Buckner, J.; Jeong, J. C.; Qi, Y.; Jo, S.; Pande, V. S.; Case, D. A.; Brooks, C. L., 3rd; MacKerell, A. D., Jr.; Klauda, J. B.; Im, W. CHARMM-GUI Input Generator for NAMD, GROMACS, AMBER, OpenMM, and CHARMM/OpenMM Simulations Using the CHARMM36 Additive Force Field. $J$. Chem. Theory Comput. 2016, 12, 405-13.

10. Huang, J.; MacKerell, A. D., Jr. CHARMM36 all-atom additive protein force field: validation based on comparison to NMR data. J. Comput. Chem. 2013, 34, 2135-45.

11. Kästner, J. Umbrella sampling. Wiley Interdiscip. Rev. Comput. Mol. Sci. 2011, 1, 932-942.

12. Hub, J. S.; de Groot, B. L.; van der Spoel, D. g_wham-A Free Weighted Histogram Analysis Implementation Including Robust Error and Autocorrelation Estimates. J. Chem. Theory Comput. 2010, 6, 3713-3720.

13. Brylinski, M. Aromatic interactions at the ligand-protein interface: Implications for the development of docking scoring functions. Chem. Biol. Drug Des. 2018, 91, 380-390.

14. Sinnokrot, M. O.; Valeev, E. F.; Sherrill, C. D. Estimates of the ab initio limit for pi-pi interactions: the benzene dimer. J. Am. Chem. Soc. 2002, 124, 10887-93. 